\title{
Sensorless Speed Control of Induction Motor Derives Using a Robust and Adaptive Neuro-Fuzzy Based Intelligent Controller
}

\author{
Abolfazl Vahedi \\ Farzan Rashidi \\ Electric Machines Research Laboratory, Iran University of Science and Technology, Tehran, Iran
}

\begin{abstract}
In this paper a novel sensorless adaptive neurofuzzy speed controller for induction motor derives is formulated. An artificial neural network (ANN) is adopted to estimate the motor speed and thus provide a sensorless speed estimator system. The performance of the proposed adaptive neurofuzzy speed controller is evaluated for a wide range of operating conditions for induction motor. These include startup, step changes in reference speed, unknown load torque and parameters variations. Obtained results show that the proposed ANN provides a very satisfactory speed estimation under the above mentioned operation conditions and also the sensorless adaptive neurofuzzy speed controller can achieve very robust and satisfactory performance and could be used to get the desired performance levels. The response time is also very fast despite the fact that the control strategy is based on bounded rationality.
\end{abstract}

\section{Introduction}

Variable speed motor drives play an important role in modern industries because they are utilized extensively in factory automation to store energy or to meet stringent load requirements. The use of variable speed motor drives is ever increasing and will maintain its momentum for several decades to come [1]. Among all different kinds of electric motor drives, the induction motor has become the subject of a large body of research in the field of electric motor drives. This is partly because the motor has an intrinsically simple and rugged structure and low manufacturing cost. Moreover, induction motor drives have the wide speed range, high efficiencies, and robustness [2]. All these merits make the motor a good candidate for the industrial applications.

Sensorless control of induction motor drives is now receiving wide attention. The main reason is that the speed sensor spoils the ruggedness and simplicity of induction motor. In a hostile environment, speed sensors can not even be mounted. However, due to the high order and nonlinearity of the dynamics of an induction motor, estimation of the angle speed and rotor flux without the measurement of mechanical variables becomes a challenging problem [3]. The advantages of speed sensorless induction motor derives are reduced hardware complexity and lower cost, reduce size of derive machine, eliminate of sensor cable, better noise immunity, increasing reliability and less maintenance requirements [4]. Various speed control algorithms for induction motor derives have been devised in the literature. Among them, PID controllers [5], optimal [6], nonlinear $[7,8]$ and robust $[9,10]$ control strategies, and neural and/or fuzzy $[3,11]$ approaches are to be mentioned. The purpose of this paper is to suggest another control approach, based on adaptive neurofuzzy controller to achieve faster response with reduced overshoot and rise time. Although the proposed method is a mathematical model based approach, it demonstrates some robustness towards model parameter variations due to the fact that the adaptive neurofuzzy based intelligent controllers are usually robust [12]. 
Furthermore a multilayer perceptron (MLP) neural network with error bakpropagation learning algorithm is designed to estimate velocity in the whole speed range satisfactorily which will be detailed in the next sections. To evaluate the usefulness of the proposed method, some simulations are performed, obtained results show that the proposed method not only is robust in the presence of the parameter variations but also its response time is very fast despite the fact that the proposed control strategy is based on bounded rationality. The structure of this paper is as follows:

Section 2 describes the induction motor and its mathematical model. In section 3, the whole structure of the proposed MLP neural network for speed estimation of induction motor is shown. Section 4 describes the structure of neuro-fuzzy controller and its application in speed control of induction motor. The performance of the proposed method under the whole range of operation conditions of induction motors is then shown in Section 5. Finally some conclusion and remarks is discussed in section 6 .

\section{Mathematical Model of Induction motors}

Many schemes based on simplified motor models have been devised to sense the speed of the induction motor from measured terminal quantities for control purposes. In order to obtain an accurate dynamic representation of the motor speed, it is necessary to base the calculation on the coupled circuit equations of the motor [13]. Since the motor voltages and currents are measured in a stationary frame of reference, it is also convenient to express these equations in that stationary frame [13]. From the stator voltage equations in the stationary frame it is obtained [14]:

$$
\begin{aligned}
\frac{d \lambda_{q r}}{d t} & =\frac{L_{r}}{L_{m}}\left(V_{q s}-\left(R_{s}+\sigma L_{s} \frac{d}{d t}\right) i_{d s}\right) \\
\frac{d \lambda_{d r}}{d t} & =\frac{L_{r}}{L_{m}}\left(V_{d s}-\left(R_{s}+\sigma L_{s} \frac{d}{d t}\right) i_{q s}\right)
\end{aligned}
$$

Where $\lambda$ is the flux linkage; $L$ is the inductance; $\quad V$ is the voltage; $R$ is the resistance; $i$ is the current, and $\sigma=1-\frac{L_{m}^{2}}{L_{r} L_{s}}$ is the motor leakage coefficient. The subscripts $r$ and $s$ denotes the rotor and stator values respectively refereed to the stator, and the subscripts $d$ and $q$ denote the $d q$-axis components in the stationary reference frame. The rotor flux equations in the stationary frame are [14]:

$$
\begin{aligned}
& \frac{d \lambda_{d r}}{d t}=\frac{L_{m}}{\tau_{r}} i_{d s}-\omega_{r} \lambda_{q r}-\frac{1}{\tau_{r}} \lambda_{d r} \\
& \frac{d \lambda_{q r}}{d t}=\frac{L_{m}}{\tau_{r}} i_{q s}+\omega_{r} \lambda_{d r}-\frac{1}{\tau_{r}} \lambda_{q r}
\end{aligned}
$$

Where $\omega_{r}$ is the rotor electrical speed and $\tau_{r}=\frac{L_{r}}{R_{r}}$ is the rotor time constant. The synchronous frequency in stationary frame is defined as follows [14]:

$\omega_{e}=\frac{\lambda_{d r} \frac{d \lambda_{q r}}{d t}-\lambda_{q r} \frac{d \lambda_{d r}}{d t}}{\lambda_{d r}^{2}+\lambda_{q r}^{2}}$

Substituting the equation (2) in the equation (3) it is obtained:

$$
\omega_{e}=\omega_{r}-\frac{L_{m}}{\tau_{r}}\left(\frac{\lambda_{d r} i_{q s}-\lambda_{q r} i_{d s}}{\lambda_{d r}^{2}+\lambda_{q r}^{2}}\right)
$$

Then substituting the equations (3) in the equation (4), and finding $\omega_{r}$ we obtain [14]:

$\omega_{r}=\frac{1}{\lambda_{d r}^{2}+\lambda_{q r}^{2}}\left[\begin{array}{l}\lambda_{d r} \frac{d \lambda_{q r}}{d t}-\lambda_{q r} \frac{d \lambda_{d r}}{d t} \\ -\frac{L_{m}}{\tau_{r}}\left(\lambda_{d r} i_{q s}-\lambda_{q r} i_{d s}\right)\end{array}\right]$

Therefore, given a complete knowledge of the motor parameters, the instantaneous speed $\omega_{r}$ can be calculated from the equation (5) where the stator currents and voltages are known along with the machine parameters, and the rotor flux linkages are obtained from equation (1). But because of the existing some derivation and integration terms, this technique, specially in real time applications, is limited in its usefulness and is not very general. To overcome these difficulties the following idea was used in this paper: With substituting the equations (1) and (2) in the 
(5) and doing some simplifications and discretizations we can obtain the motor speed $\omega_{r}$ as a functions of the stator voltages and currents as follows:

$$
\omega_{r}=f\left(\begin{array}{l}
V_{s}(k), V_{s}(k-1), V_{s}(k-2) \\
i_{s}(k), i_{s}(k-1), \ldots, i_{s}(k-3)
\end{array}\right)
$$

So it is possible to estimate the speed of an induction motor in the whole speed range in another fashion by solving the equation (6) using an adaptive neural network (NN). Here a multilayer perceptron (MLP) neural network with error bakpropagation learning algorithm is designed to estimate velocity.

\section{Speed Estimation of Induction Motors Using NN}

Neural Networks are a family of intelligent algorithms which can be used for time series prediction, classification, control and identification. Neural networks have an ability to train with various parameter of induction motor. As a nonlinear function, they can be used for identifying the extremely nonlinear system parameters with high accuracy. Recently, the use of neural networks to identify and control nonlinear dynamic systems has been proposed because they can approximate a wide range of nonlinear functions to any desired degree of accuracy. Moreover, they have the advantages of extremely fast parallel computation, immunity from input harmonic ripples, and fault tolerance characteristics [15]. Also there have been some investigations into the application of NNs to power electronics and ac drives, including speed estimation. This technique gives a fairly good estimate of the speed and is robust to parameter variation [16]. However, the neural network speed estimator should be trained sufficiently with various patterns to get good performance. In this paper, a new speed estimation method of an induction motor is proposed. This method is based on the multilayer backpropagation neural network Back propagation algorithm is one of the most popular algorithms for training a network due to its success from both simplicity and applicability viewpoints. The algorithm consists of two phases. Training phase and recall phase. In the training phase, first, the weights of the network are randomly initialized. Then, the output of the network is calculated and compared to the desired value. In sequel, the error of the network is calculated and used to adjust the weights of the output layer. The generalized delta rule of MLP neural network is as follows [17]:

$$
\Delta w_{j i}(n)=\alpha \Delta w_{j i}(n-1)+\eta \delta_{j}(n) y_{i}(n)
$$

Where $\eta$ is the learning rate, $\alpha$ is the momentum parameter, $\delta_{j}$ is the local gradient of neuron $j, y_{i}$ is the output signal of neuron $i$ and $\Delta w_{j i}(n)$ is the weight correction connecting neuron $i$ to neuron $j$. The basic problem in training a NN to recognize induction motor speed is that the functional relationship between the speed and stator parameters. As seen in equation (6) if the following terms are considered as inputs to $\mathrm{NN}$, it should be able to estimate the speed with high accuracy:

$V_{s q}(k), V_{s q}(k-1), V_{s q}(k-2), V_{s d}(k), V_{s d}(k-1)$
$V_{s d}(k-2), i_{s q}(k), i_{s q}(k-1), i_{s q}(k-2), i_{s q}(k-3)$
$i_{s d}(k), i_{s d}(k-1), i_{s d}(k-2)$ and $i_{s d}(k-3)$

As seen from equation (8) fourteen nodes are needed at the input layer. The computation time of the $\mathrm{NN}$ increases dramatically with the numbers of the nodes. Since all needed information for speed estimation is provided by equation (8), so we can use the MLP neural network consists of three layers with one hidden layer. The sigmoid functions are used at the hidden layer and the linear function is used at the output layer. Figure 1 shows the internal structure of the NN. With assumption that the stator currents and voltages are known along with the machine parameters, the training algorithm of the $\mathrm{NN}$ speed estimator will be as follows.

1- Initially randomize the MLP NN weights between -1 to +1 .

2- Obtain the stator voltages with two delays and currents with three delays.

3- Calculate the error between target, $\omega_{r}(k)$

and estimated output, $\hat{\omega}_{r}(k)$.

4- Adjust the weights of the NN.

5-Calculate the output of the NN, $\hat{\omega}_{r}(k)$ 


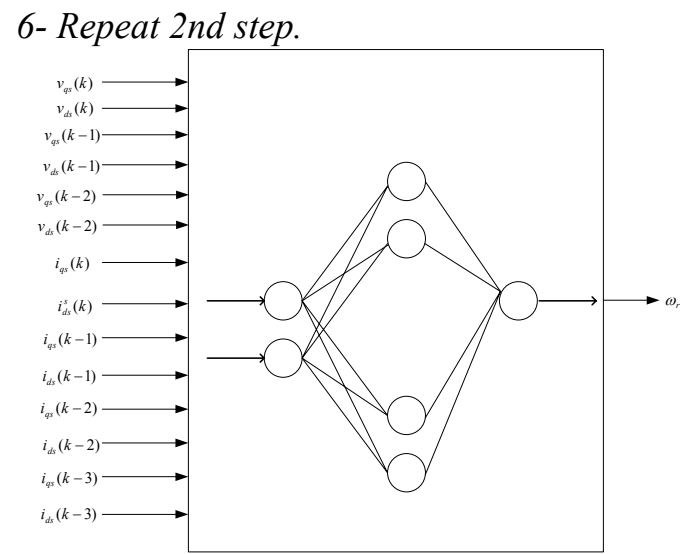

Figure 1. The internal structure of the proposed NN.

\section{4- Design of Sensorless Speed Adaptive Neurofuzzy Controller for induction motors}

Neural networks and fuzzy logic are two complimentary techniques. Neural networks can learn from data; however, understanding the knowledge learned by neural networks has been difficult. To be more specific, it is usually difficult to develop an insight about the meaning associated with each neuron and each weight. Hence, neural networks are often viewed as a black box approach-we know what the box does, but not how the box does [18]. In contrast, fuzzy rule based model are easy to be understood because it uses linguistic terms and the structure of IF-THEN rules. Unlike neural networks, however, fuzzy logic by itself can not learn. The learning and identification of fuzzy logic systems need to adopt techniques from other areas, such as statistics, system identification. Since neural networks can learn, it is natural to marry these two techniques [17]. This marriage has created a new term-fuzzy neural networks (FNN). An FNN can be loosely defined as a system that uses a combination of fuzzy logic and neural networks. Two major approaches of trainable neurofuzzy models can be distinguished. The network based Takagi-Sugeno fuzzy inference system, which is used here, and the locally linear neurofuzzy model. It is easy to see that the locally linear model is equivalent to Takagi-Sugeno fuzzy model under certain conditions, and can be interpreted as an extension of normalized RBF network as well. The proposed adaptive neuro-fuzzy controller is a kind of unsupervised learning methods for autonomous agents to acquire action rules to adapt cue of reinforcemental reward and punishment. In this method the teacher of conventional supervised learning is replaced by an intelligent critic that assesses the performance of controller and evaluates the current states of system and generates proper reinforcement signal $r$. The controller should modify its characteristics so that the critic stress $(r)$ is decreased. The performance of the critic can be compared with the performance of emotional hue in humans. In absence of an exact evaluation of the present state in term of the objective value function, reinforcement cues like stress, satisfaction and etc. can be guide our control action into changing in the right direction so as to produce desired response. Similarly, the critic evaluates the state of system and generates a signal called reinforcement signal (r). This signal is used to train and fine tune the main controller. Basically this critic acts as intelligent guide for the controller. The learning mechanism will be adapted the controller in order to satisfy critic and reduce its stress. This is a key idea of the proposed method in its using at the control systems. The structure of the adaptive neuro fuzzy controller is illustrated in figure 2. The mathematical description of this method is as follows:

The Takagi-Sugeno fuzzy inferendNPPJ্T is based on fuzzy rules of the following type:R Rule $_{i}:$ If $u_{1}=A_{i 1}$ And ... And $u_{p}=A_{i p}$ (9) then $y=f_{i}\left(u_{1}, u_{2}, \ldots, u_{p}\right)$

Where $i=1 \ldots M$ and $M$ is the number of fuzzy rules. $u_{1}, \ldots, u_{p}$ are the inputs of network, each $A_{i j}$ denotes the fuzzy set for input $u_{j}$ in rule $i$ and $f_{i}($.$) is a crisp function$ which is defined as a linear combination of inputs in most applications

$y=\omega_{i 0}+\omega_{i 1} u_{1}+\omega_{i 2} u_{2}+\mathrm{K}+\omega_{i p} u_{p}$

Thus the output of this model can be calculated by 


$$
y=\frac{\sum_{i=1}^{M} f_{i}(\underline{u}) \mu_{i}(\underline{u})}{\sum_{i=1}^{M} \mu_{i}(\underline{u})}, \mu_{i}(\underline{u})=\prod_{j=1}^{p} \mu_{i j}\left(u_{j}\right)
$$

A simple form of $f_{i}(u)$ can be as

$$
f_{i}(u)=a_{i} u_{1}+b_{i} u_{2}+c_{i}
$$

The out put of controller is in the following form:

$$
y=\frac{\sum_{i=1}^{n} \mu_{i}\left(a_{i} u_{1}+b_{i} u_{2}+c_{i}\right)}{\sum_{i=1}^{n} \mu_{i}}
$$

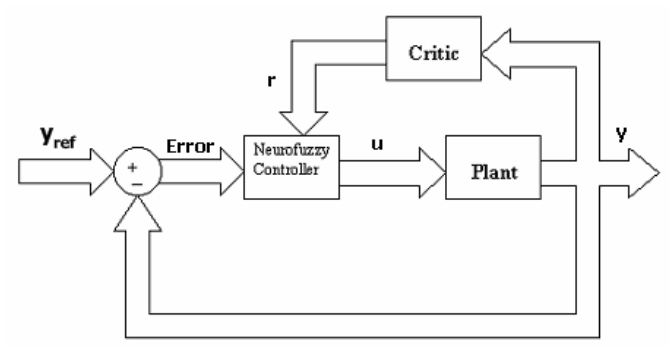

Figure 2- Block Diagram of adaptive neurofuzzy controller

Where $n$ is number of controller fuzzy rules, $\mu_{i}$ is the firing strength of $i$ th rules, $u_{1}$ is the first and $u_{2}$ is the second one for two input type controller. In this paper we choose $u_{1}=e$ (speed error) and $u_{2}=\&$ (speed error derivative). The neurofuzzy controller applied in here, as mentioned above, is a standard Sugeno fuzzy controller composed of four layers. In the first layer, all inputs are mapped into the range of $[-1,+1]$. In the second layer, the fuzzification process is performed using Gaussian membership functions with seven labels for each input. In layer 3, decision-making is done using MaxProduct law and defuzzification is carried out in the fourth layer in order to calculate the proper control signal using (13). $a_{i}, b_{i}, c_{i}$ are parameters to be determined via learning mechanism. Although the proposed FNN seems to have the same architecture with pure neural networks, they differ in two major ways:

$A$. The nodes and links in an FNN are usually comprehensible and perform different operation because each of them corresponds to a specific component in fuzzy system.

$B$. The nodes in an FNN are usually not fully connected to those in adjacent layers because the connections reflect the rule structure of the fuzzy system.

The main purpose in the adaptive neurofuzzy based intelligent controller is to optimization an energy function. This aim can be extracted trough bellow energy function:

$E=\frac{1}{2} k r^{2}$

Where $k$ is a positive constant and $r$ is a reinforcement signal. By minimizing this energy function, we can reduce the total stress of the system and satisfy all critics. With applying Newton gradient decent method the changes in weight must be followed by bellow general rule:

$$
\Delta w_{i}=-\eta \frac{\partial E}{\partial w_{i}}
$$

Where $\eta$ is the learning rate of the corresponding neurofuzzy controller. In order to calculate the RHS of (15), the chain rule is used:

$\Delta w_{i}=-\eta \frac{\partial E}{\partial r} \cdot \frac{\partial r}{\partial y} \cdot \frac{\partial y}{\partial u} \cdot \frac{\partial u}{\partial w_{i}}$

From (14),

$\frac{\partial E}{\partial r}=k r$

And also

$e=y_{\text {ref }}-y$

Then $\frac{\partial r}{\partial y}=-\frac{\partial r}{\partial e}$

Since with the incrimination of error, $r$ will also be incremented and on the other hand, on-line calculation of $\frac{\partial r}{\partial e}$ is accompanied with measurement errors, thus producing unreliable results, we have

$$
\frac{\partial r}{\partial y}=-\frac{\partial r}{\partial e}=-\lambda \quad(\lambda>0)
$$

Which in our calculations only the sign of it $(-1)$ is used. Also $\frac{\partial y}{\partial u}=J$, where $J$ is a Jacobean Matrix of the system. From (15) to (20), $\Delta w_{i}$ will be calculated as follows: 


$$
\Delta w_{i}=\eta k r J \cdot \frac{\partial u}{\partial w_{i}}
$$

Equation (21) is used for updating the learning parameters $a_{i}, b_{i}$ and $c_{i}$ in (13), which is straightforward:

$$
\begin{gathered}
a_{i \text { New }}=a_{\text {iOld }}+\eta r e \frac{u_{i}}{\sum_{i=1}^{n} u_{i}} \\
b_{\text {iNew }}=b_{\text {iOld }}+\eta r \& \frac{u_{i}}{\sum_{i=1}^{n} u_{i}} \\
c_{i \text { New }}=c_{\text {iOld }}+\eta r \frac{u_{i}}{\sum_{i=1}^{n} u_{i}}
\end{gathered}
$$

Fuzzy systems are very useful for critic modeling because it just gives us an

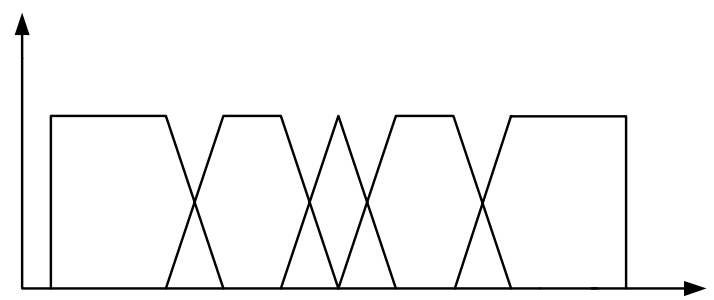

Figure 3. Error and rate error input fuzzy sets membership function

Figures 3 and 4 show the membership function sets for the inputs and output.

Table 1: Rule base of the reinforcement signal $r$

\begin{tabular}{|c|c|c|c|c|c|}
\hline $\begin{array}{c}\mathrm{e} \rightarrow \\
\Delta \mathrm{e} \vee\end{array}$ & NL & NS & ZE & PS & PL \\
\hline PL & ZE & PS & PM & PL & PL \\
\hline PS & NS & ZE & PS & PM & PL \\
\hline ZE & NM & NS & ZE & PS & PM \\
\hline NS & NL & NM & NS & ZE & PS \\
\hline NL & NL & NL & NM & NS & ZE \\
\hline $\begin{array}{l}\text { PL: Positive Large } \\
\text { PM: Positive Medium } \\
\text { PS: Positive Small } \\
\text { ZE: Zero }\end{array}$ & \multicolumn{3}{|c|}{$\begin{array}{c}\text { NS: Negative Small Negative Medium } \\
\text { NL: Negative Large }\end{array}$} \\
\hline
\end{tabular}

approximate evaluation of current states of system. So we define it as fuzzy form. In this paper the inputs of critic have been defined as error signal and its derivative. The output of critic is a signal between $[-1,1]$ which shows the system performance. If this signal becomes zero, it means that the critic is satisfied by the performance of controller. Otherwise when it becomes larger, it shows the more stress and more dissatisfaction. Fuzzification and defuzzification have been performed by Gaussian membership functions with 5 labels for each input and 7 labels for the output; deduction is performed by Max-Product law and for defuzzification the centroid law is used.

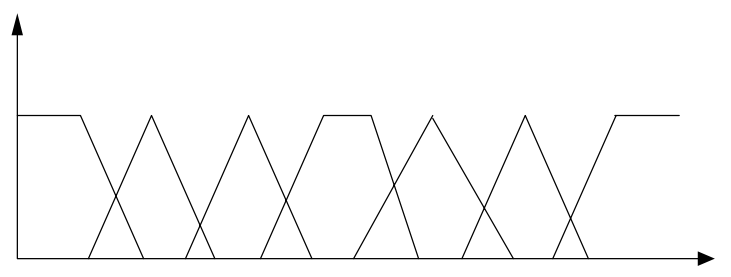

Figure 4. Output fuzzy sets membership function

The fuzzy sets and rules base of this critic is shown in table 1. Figure 5 shows the Block diagram of speed-sensorless controller using adaptive neurofuzzy controller.

\section{Simulation Results}

In this section we illustrate the performance of the proposed neural network based sensorless adaptive fuzzy-neural controller by simulations using the MATLAB software package. The induction motor considered in this paper has the following data and parameters:

3 phase, $380 \mathrm{~V}, 15 \mathrm{KW}, 31 \mathrm{~A}$, 2 poles ,2895rpm

$$
\begin{gathered}
R_{r}=1.46 \Omega, R_{s}=0.603 \Omega, L_{l r}=4.72 \mathrm{mH}, \\
L_{l s}=4.72 \mathrm{mH}, L_{m}=330.2 \mathrm{mH}
\end{gathered}
$$

As we mentioned before, we only use the speed error and its derivation as the inputs to the adaptive fuzzy scheme, each with seven 
fuzzy sets on their ranges respectively. Because the states are two, the total numbers of fuzzy rules are 49. Using the algorithm developed in the previous sections, the dynamic performance of the observer-based sensorless adaptive fuzzy speed controller has been simulated for a wide range of operating conditions. The results of these tests will be detailed below. In addition, the important effects of parameter variations are examined in detail in order to verify the robustness of the system. In first simulation the speed command is set as $220 \mathrm{rad} / \mathrm{s}$.

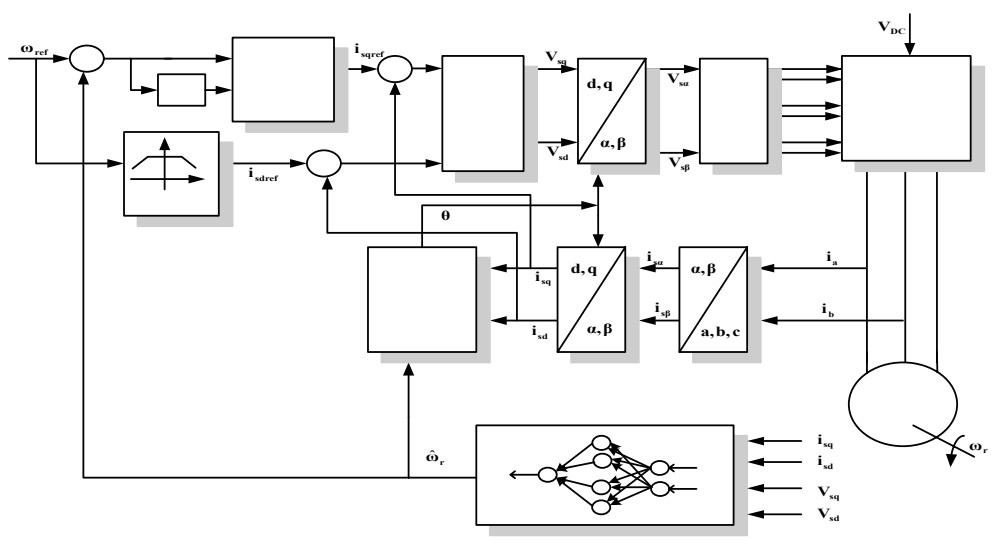

Figure 5: Block diagram of speed-sensorless controller using adaptive neurofuzzy controller

The speed response when the induction motor is started from standstill has been given in Fig. 6. As seen the settling time for the estimated speed to track the speed command is less than $0.1 \mathrm{sec}$. Furthermore, the steady state error is almost zero without noticeable

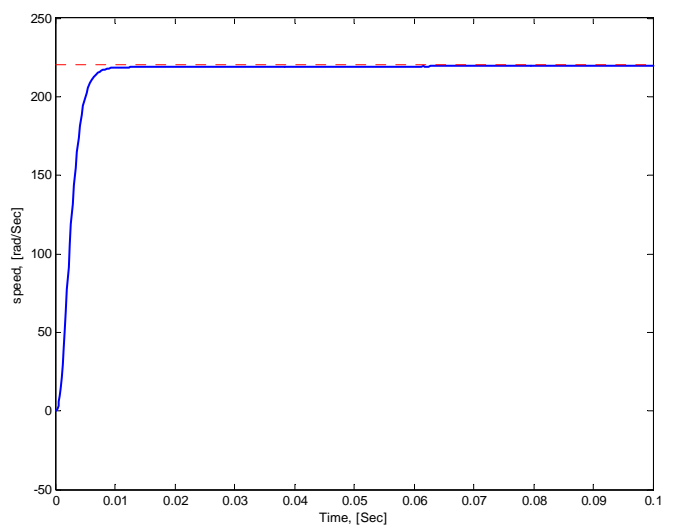

Fig.6. Speed response when speed command is set as $220 \mathrm{rad} / \mathrm{s}$

We evaluated the performance of the adaptive neurofuzzy controller with respect to step changes in reference speed. In this test, the motor is started from zero speed with a speed command of $100 \mathrm{rad} / \mathrm{s}$. The speed command changes to $200 \mathrm{rad} / \mathrm{s}$ at $t=0.1 \mathrm{sec}$ and speed ripples. The speed response when the speed command is set as $70 \mathrm{rad} / \mathrm{s}$ is given in Fig. 7. As seen from this figure the obtained result is satisfactory in terms of settling time, overshot and rise time.

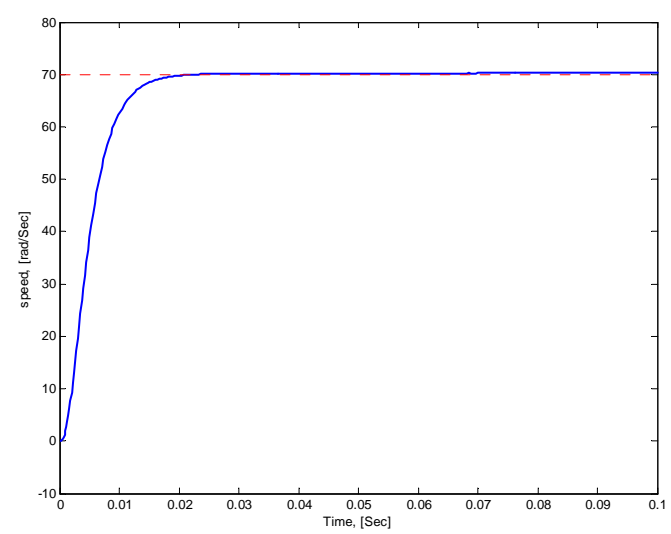

Fig. 7. Speed response when speed command is set as $70 \mathrm{rad} / \mathrm{s}$

$150 \mathrm{rad} / \mathrm{s}$ at $t=0.2 \mathrm{sec}$, finally the speed command changes to $50 \mathrm{rad} / \mathrm{s}$ at $t=0.3 \mathrm{sec}$. Fig. 8 shows the behavior of the proposed controller under these step changes in speed command. It can be seen that the motor speeds exhibit smooth performance under 
different reference speed, and a rapid transient response. Therefore in this test it is shown clearly that the adaptive neurofuzzy speed controller can track variable reference speed rapidly and smoothly. It is common that in many cases the induction motor may be operated with an unknown load torque. Thus, the performance of the developed scheme under unknown load torque is examined here. In this test, the external load torque is set to $5 \mathrm{Nm}$ during $0-0.2 \mathrm{sec}, 7 \mathrm{Nm}$ during $0.2-0.4 \mathrm{sec}$, and $9 \mathrm{Nm}$ during $0.4-$

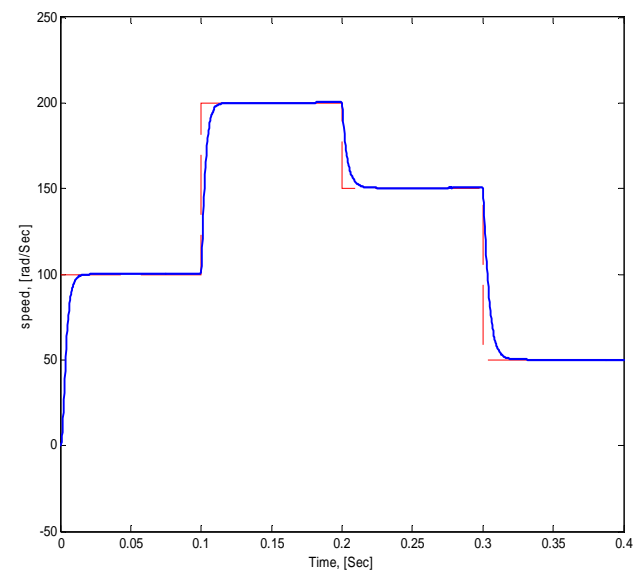

Fig. 8. Speed response when speed command is changed

Now we test the robustness of the adaptive fuzzy-neural controller toward modeling errors. The mechanical parameters $(J, B)$ can not always be obtained accurately and they may also vary during the induction motor operation. However, the uncertainties on the mechanical parameters can be well handled by the process of load torque estimation [3]. Thus in this section only the test under electrical parameter variations is done here.

We illustrate the robustness toward electrical parameters by using rotor resistance as an example. During the induction motor operation, $R_{r}$ will increase due to temperature rise. At the beginning of this test, the measured $R_{r}=1.46 \Omega$. Suppose this value changes to $R_{r}=2.46 \Omega$ when $t=0.1 \mathrm{sec}$. The speed command is $100 \mathrm{rad} / \mathrm{s}$. The result of Fig. 10 shows the robustness against this resistance variation. It is shown in Fig. 10 that the estimated speed can still track the
$0.6 \mathrm{sec}$. The reference speed is $100 \mathrm{rad} / \mathrm{s}$ and the initial estimated load torque is set as $0 \mathrm{Nm}$. In Fig. 9 we can see the speed responses under these large step changes in the load torque. We can see even with such fast changes in load the observer-based controller also exhibits very good performance with a fast response. Hence, the results above have shown the robustness of the adaptive neurofuzzy speed controller toward variable load torque, which is the common case in practice.

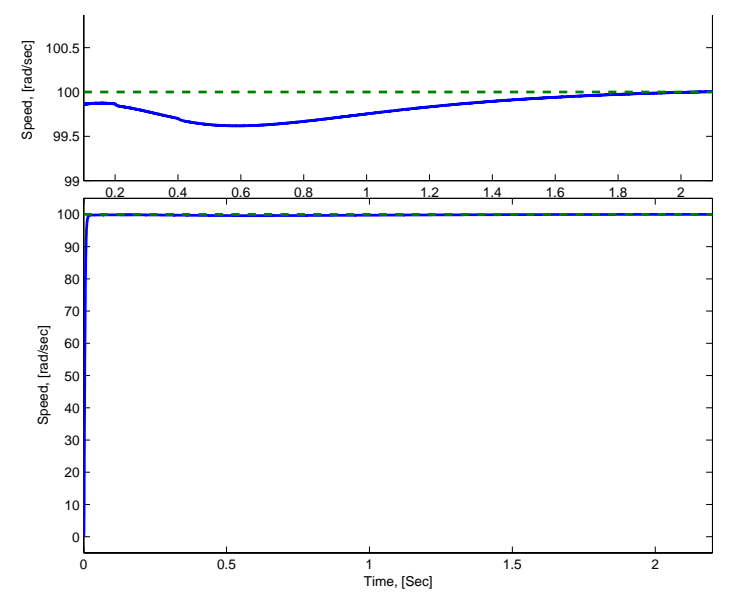

Fig. 9. Speed response when the external load torque is changed reference speed very well without noticeable deviation.

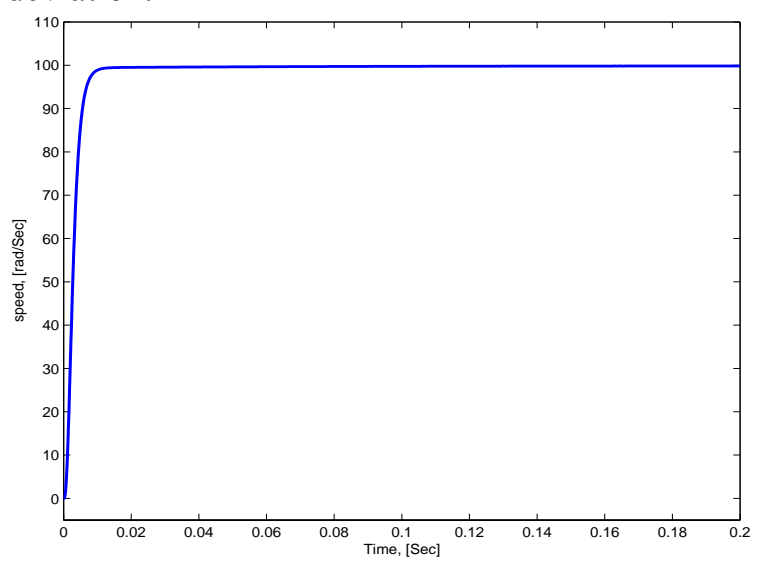

Fig. 10. Speed response when the rotor resistance was changed at $\mathrm{t}=0.1 \mathrm{sec}$

Hence in this test, we demonstrated that the developed NN-based sensorless adaptive fuzzy-neural network is robust to the electrical parameter variations. Meanwhile, 
the mechanical parameter variations can be well handled by the proposed method as if they were due to load torque variations, which have been shown in the above. 6 .

\section{Conclusion}

In this paper, a novel adaptive neurofuzzy controller based rotor speed estimation algorithm was presented for induction motor drives. Furthermore a multilayer perceptron (MLP) neural network with error bakpropagation learning algorithm was designed to estimate velocity in the whole speed range to provide a sensorless speed estimator system. To demonstrate the effectiveness and applicability of the proposed neurofuzzy controller based speed estimation algorithm, simulation results under the whole range of the operation conditions was presented, including low and high speed, transient conditions, and parameters variations as well. In conclusion, the ANF controller based scheme presented a very good performance under the whole range of operation conditions; include startup, low and high speed, low and high acceleration. Moreover it was also robustness to the parameters variations.

\section{References}

[1]- Rashidi. F., C. Lucas, "Applying CBBEL (Context Based Brain Emotional Learning) to SR Motors", WSEAS Transactions on Systems, 2003, 1082-1086.

[2]- Bottura, C.P., Neto, M.F.S.; Filho, S.A.A., "Robust speed control of an induction motor: an /spl Hscr/sub /spl infin/ control theory approach with field orientation and /spl mu/-analysis", IEEE Transactions on Power Electronics, Vol. 15, pp. 908-915, 2000

[3]- Rashidi, F., Rashidi, M., "Design of robust sliding mode speed control with fuzzy approach for induction motors", IEEE International Conference on Industrial Technology, ICIT03, 2003, pp. 27-30

[4]- Joachim Holtz, "Sensorless speed and position control of induction motor derives", IEEE international conference on Industrial Electronics, IECON03, 2003, USA

[5]- Takano, A.; "Quick-response torquecontrolled induction motor drives using phaselocked loop speed control with disturbance compensation", IEEE Transactions on Industrial
Electronics, Volume: 43 Issue: 6, pp. 640 -646, 1996

[6]- Negm, M. M. M.; "Torque optimized speed control of a 3-phase induction motor", IEEE International Conference on Power System Technology, Volume: 1, pp. 67-72, 2000

[7]- V., I., Utkin, "Sliding mode control design principles and applications to electric drives," IEEE Trans. Ind. Appl., vol. 40, Feb. 1993.

[8]- E. Y. Y. Ho and P. C. Sen, "A microcontroller-based induction motor drive system using variable structure strategy with decoupling," IEEE Trans. Ind. Electron., vol. 37, June 1990.

[9]- Guang Feng; Lipei Huang; Dongqi Zhu;"A robust controller for improving disturbance rejection in speed control of induction motors", International Conference on Power Electronics and Drive Systems, Vol. 1, pp. 432-437, 1999

[10]- C. Schauder, "Adaptive speed identification for vector control of induction motor without rotational transducers," IEEE-IAS., 1989, pp. 493-499.

[11]- I. Ben-Brahim and T. Kudor, "Implementation of an induction motor speed estimator using neural networks," in Proc. IPEC, 1995, pp. 52-57.

[12]- Rashidi, F., Online short term load forecasting in power systems using emotional critic based fuzzy approach", LNAI journal, 2004 [13]- Ho-Seok Lee; Taeck-Kie Lee; Soon-Bong Cho; Dong-Seok Hyun, "Speed control of induction motor using fuzzy algorithm with hierarchical structure", IEEE Conf., TENCON '93, vol.5, 551-554, 1993

[14]- Bose, B.K., 2001, Modern Power Electronics and AC Drives., Prentice Hall, New Jersey.

[15]- Rashidi, F., M.Rashidi, "Robust and Adaptive Tuning of Power System Stabilizers Using Artificial Neural Networks", lecture notes in artificial intelligence journal, 2004

[16]- Huang, S.J., Huang, C.L. and Lin, Y.S., 1998, Sensorless speed identification of vector controlled induction drives via neural network based estimation, Electric Power System Research, 48, 1-10.

[17]- P. K. Simpson, "Artificial neural systems: Foundations, Paradigms, Applications, and Implementations", Pergamon, Elmsford, NY, 1990.

[18]- J. Stanley, "Introduction to neural networks", California Scientific Software, Siera Madre, CA, 3rd edition, 1990 